\title{
Frontal operculum gliomas: language outcome following resection
}

\author{
John D. Rolston, MD, PhD, ${ }^{1}$ Dario J. Englot, MD, PhD, ${ }^{1}$ Arnau Benet, MD, ${ }^{1}$ Jing Li, MD, MPhil, ${ }^{1}$ \\ Soonmee Cha, MD, ${ }^{2}$ and Mitchel S. Berger, MD' \\ 1Department of Neurological Surgery, and 'Department of Radiology and Biomedical Imaging, University of California, San \\ Francisco, California
}

\begin{abstract}
OBJECT The dominant hemisphere frontal operculum may contain critical speech and language pathways, and due to these properties, patients with tumors of the opercular region may be at higher risk for postoperative speech dysfunction. However, the likelihood of incurring temporary or permanent language dysfunction is unknown.

METHODS The authors retrospectively analyzed their cohort of patients with frontal gliomas to identify those tumors that predominantly involved the dominant frontal operculum. Each tumor was classified as involving the pars orbitalis, pars triangularis, pars opercularis, or a combination of some or all of these areas. The authors then identified and compared characteristics between those patients experiencing transient or permanent speech deficits, as opposed to those with no language dysfunction.
\end{abstract}

RESULTS Forty-three patients were identified for inclusion in this analysis. Transient deficits occurred in 12 patients $(27.9 \%)$, while 4 patients (9.8\%) had persistent deficits involving language. Individuals with preoperative language deficits and patients with seizures characterized by speech dysfunction appear to be at the highest risk to develop a deficit (relative risks 3.09 and 1.75, respectively). No patient with a tumor involving the pars orbitalis experienced a persistent deficit.

CONCLUSIONS Resection of gliomas is widely recognized as a critical element of improved outcome. Given the low rate of language morbidity reported in this group of patients, resection of gliomas within the dominant frontal operculum is well-tolerated with acceptable morbidity and, in this particular location, should not be a deterrent in the overall management of these tumors.

http://thejns.org/doi/abs/10.3171/2014.11.JNS132172

KEY WORDS operculum; Broca's area; pars opercularis; pars triangularis; glioma; oncology

$\mathrm{T}$ HE dominant hemisphere frontal operculum, which contains Broca's area, is considered essential for language function. Numerous studies have examined lesions of the frontal operculum and correlated these lesions with clinical aphasias., ${ }^{2,3,7}$ Given the critical nature of this area, many patients with intrinsic operculum tumors are often considered high risk for resection, even though extent of tumor resection is often an important component in determining overall survival.

Despite the perceived risk of frontal operculum tumor resections, many patients will undergo more than a biopsy or partial resection under awake language mapping conditions, or asleep with or without motor mapping. These patients appear to do well when language mapping is used. For example, Kral et al. report on 11 patients with tumors in Broca's area who underwent resection with mapping, none of whom had postoperative speech deficits. ${ }^{23}$ Similarly, Plaza et al. report a patient who had a glioma removed from the left inferior and middle frontal gyri, but maintained language function. ${ }^{28}$

The frontal operculum of the inferior frontal gyrus is usually characterized as having 3 regions, the anterior pars orbitalis, the middle pars triangularis, and the posterior pars opercularis. ${ }^{34}$ Broca's area is cytoarchitectonically defined as Brodmann's areas 44 and 45 (pars opercularis and triangularis), and is believed to contain language areas critical for speech expression. Nevertheless, language-mapping studies in awake patients undergoing craniotomies show a high degree of variability. For instance, in the largest study to date (151 patients), cortical language sites were only identified in the dominant frontal lobe, including the operculum, in $60.9 \%$ of patients. ${ }^{31}$ Even when the frontal

ABBREVIATIONS AF = arcuate fasciculus; $\mathrm{Cl}=$ confidence interval; $\mathrm{EOR}=$ extent of resection; $\mathrm{GTR}=$ gross-total resection; $\mathrm{MSI}=$ magnetic source imaging; $\mathrm{RR}=$ risk ratio; SLF = superior longitudinal fasciculus; STR = subtotal resection.

SUBMITTED February 24, 2014. ACCEPTED November 13, 2014.

INCLUDE WHEN CITING Published online January 30, 2015; DOI: 10.3171/2014.11.JNS132172.

DISCLOSURE The authors report no conflict of interest concerning the materials or methods used in this study or the findings specified in this paper. 
lobe does contain critical speech sites, there continues to be high variability in location when based off anatomical landmarks, i.e., speech sites are not always within the same region of the anatomically defined frontal operculum..$^{29}$

In an attempt to characterize the risk these patients are exposed to with surgery to this area, we analyzed a cohort of patients with left-sided tumors involving the frontal operculum. By examining their baseline neurological status and clinical history, along with the results of the surgical procedure, we describe the risk profile of developing postoperative language deficits.

\section{Methods \\ Study Population}

We retrospectively reviewed 43 patients harboring tumors in the frontal operculum who underwent resection by the senior author (M.S.B.). All patients were treated at the University of California, San Francisco, Medical Center between 1998 and 2013. Patient data were extracted from a prospectively maintained database of all surgeries performed by the senior author, by manually searching for all patients with tumors in the left frontal lobe. These data were further screened for tumors within the left frontal operculum by reviewing the preoperative MR images. In the senior author's experience, language sites have never been identified in the frontal operculum greater than $3 \mathrm{~cm}$ from the prerolandic sulcus. Thus, any lesion that was to be resected at or greater than this distance was performed with the patient asleep, because awake language mapping was not necessary. In 2 cases in which an awake procedure was not conducted, despite a tumor within $3 \mathrm{~cm}$ of the prerolandic sulcus, the reasons were mass effect (making it unsafe for an awake procedure) and patient refusal to undergo an awake operation.

\section{Intraoperative Mapping}

Direct electrical stimulation was used in 33 patients to localize motor and/or language areas and to minimize surgical morbidity, as has been previously described.4-6 Briefly, for those patients undergoing awake craniotomy for speech mapping, a bipolar electrode was used to stimulate the cortex and subcortical white matter using constant-current square-wave pulses (1-msec pulse width) delivered at $60 \mathrm{~Hz}$. Stimulus intensity was initiated at 1.5 $\mathrm{mA}$ and increased to a maximum of $6 \mathrm{~mA}$ if no responses were identified. This intensity has previously been shown to be effective at preserving language function. ${ }^{31}$ For cases in which patients were under general anesthesia, currents of up to $16 \mathrm{~mA}$ were used to identify motor responses. All work was performed in accordance and with the approval of the University of California, San Francisco, Committee on Human Research.

Intraoperative photos were available for a subset of these patients, allowing us to retrospectively assess the distance of speech arrest or language sites (if present) to the resection cavity. This was performed for all 20 patients who had intraoperative photos available. Distances were determined by using the known diameter of the numbered tickets $(5 \mathrm{~mm})$ and using this measurement to determine the distance to the edge of the visible resection cavity.
All tumors were classified according to their location within the frontal operculum. The frontal operculum is subdivided into 3 regions (Fig. 1), as previously described: ${ }^{34}$ the anterior pars orbitalis (Brodmann's area 47), middle pars triangularis (Brodmann's area 45), and the posterior pars opercularis (Brodmann's area 44). Involvement of each area was characterized using complete MRI sequences.

All patients underwent preoperative MRI within 48 hours of surgery (Figs. 2 and 3). Postoperatively, all patients underwent MRI within 48 hours to characterize the extent of resection (EOR) and examine for areas of restricted diffusion. All MR images were read by neuroradiologists and further reviewed by the authors. If there was disagreement between the examining neuroradiologist and authors, the "worse" of the two readings would be used. For example, if the neuroradiologist saw no restricted diffusion, but the authors did, the case would be characterized as having restricted diffusion.

Preoperative magnetic source imaging (MSI) was available for 27 of the 43 patients, and was obtained by a whole-head 275-channel system. Verb generation and the Boston naming test were used to identify language dipoles, which were then projected onto MRI anatomical images acquired on the same date. Patients were excluded from MSI acquisition if they were unable to participate with the language tasks, if there was no plan to perform awake language mapping, or if the lesion was believed to be in the pars orbitalis and therefore had a low likelihood to impact language function.

Low- and high-grade tumors were volumetrically analyzed by measuring hyperintense regions on axial T2weighted fluid-attenuated inversion-recovery (FLAIR) and T1-weighted contrast-enhanced MR images. For each case, the tumor was segmented manually across all slices with region-of-interest analysis to compute pre- and postoperative volumes in cubic centimeters. The EOR was calculated as follows: 100 - (postoperative tumor volume/ preoperative tumor volume) $\times 100$, with $100 \%$ indicating gross-total resection (GTR) and less than $100 \%$ represent-

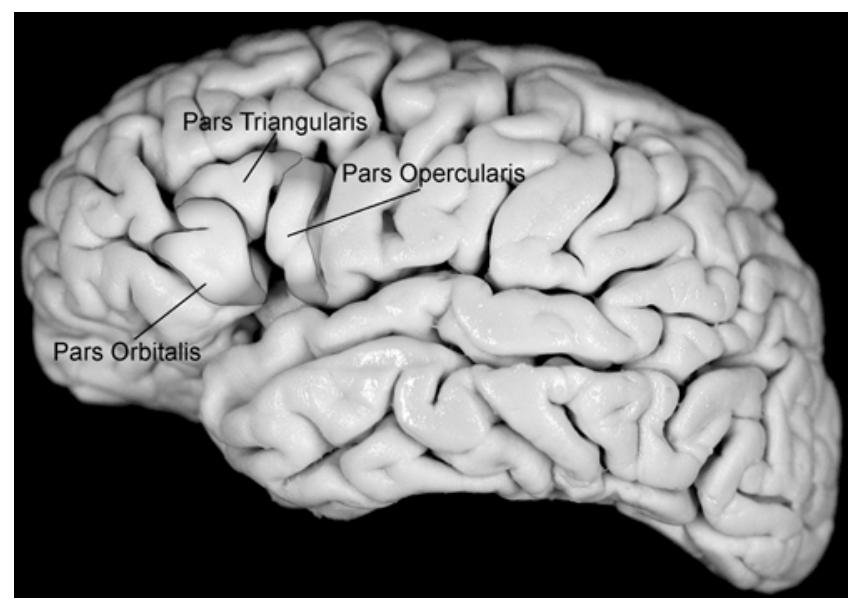

FIG. 1. Surface anatomy of the frontal operculum. This lateral view of a cadaveric brain shows the highlighted frontal operculum. The pars orbitalis, triangularis, and opercularis are labeled. 

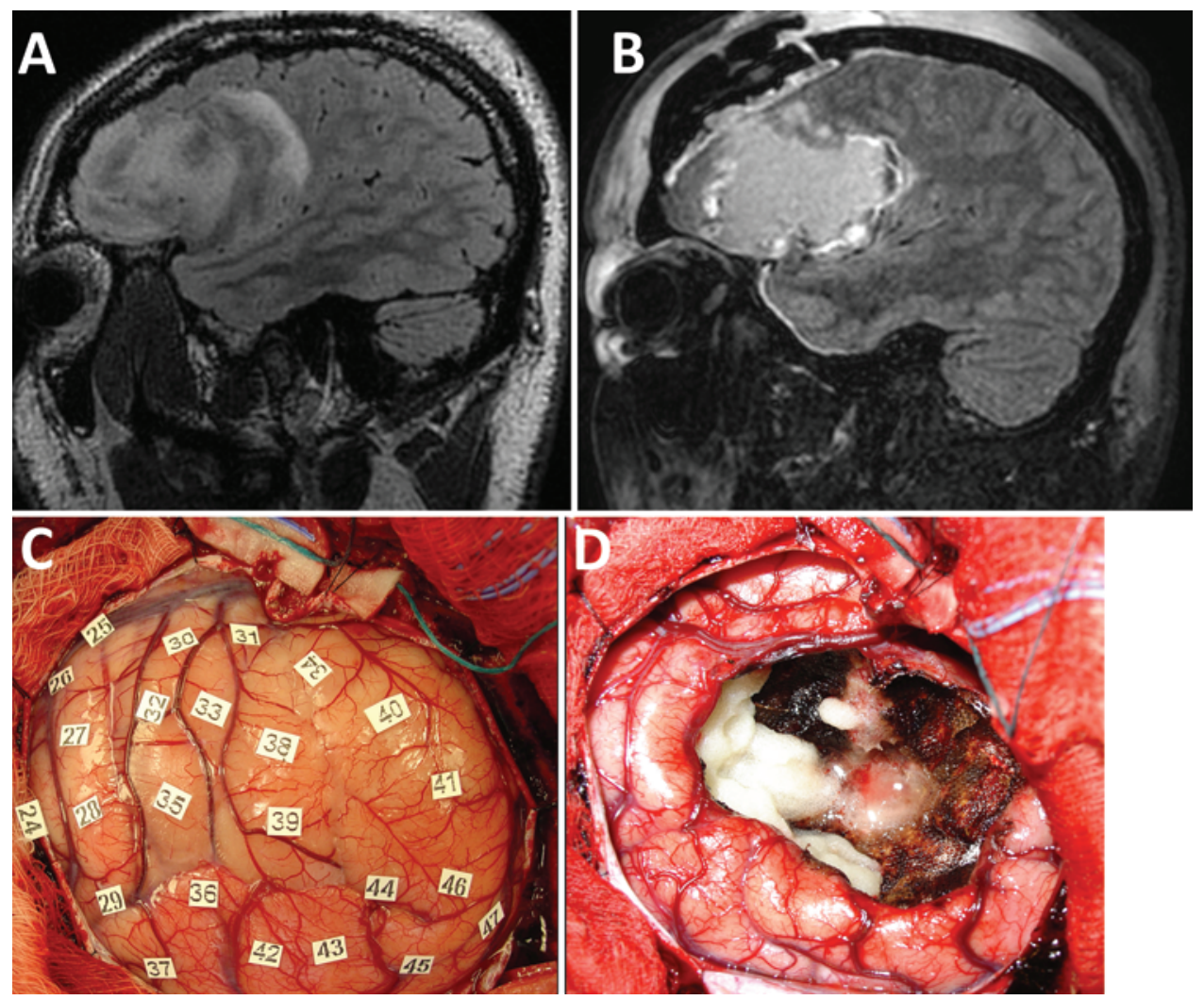

FIG. 2. Magnetic resonance images ( $A$ and $B$ ) and intraoperative photographs ( $C$ and $D)$ from a representative patient without postoperative language dysfunction. A: Preoperative parasagittal FLAIR image showing the patient's left frontal opercular oligoastrocytoma involving the pars orbitalis, triangularis, and opercularis. B: Postoperative FLAIR image showing GTR of the tumor. There was no effect on the patient's speech or language function after the operation. C: Intraoperative photograph of the operculum prior to resection and after mapping. None of the stimulated areas evoked motor movements or errors in reading or naming. D: Intraoperative photograph after the surgical resection was completed showing GTR, confirmed radiologically postoperatively. Figure is available in color online only.

ing subtotal resection (STR). Because this study did not evaluate EOR in relation to patient outcome, no further delineation in the degree of resection was determined.

Each patient was examined independently by at least two physicians both pre- and postoperatively (attending neurosurgeons and attending neurooncologists), using a standard neurological examination that included object naming, repetition, reading, counting, and calculation. When identifying language dysfunction, the poorer of the multiple neurological examinations was used. In an effort to be as conservative as possible, any degree of speech dysfunction, from word-finding difficulty to complete expressive aphasia, was counted as a deficit. Deficits were considered transient if they resolved within the first 3 months after surgery.

For anatomical photographs of subcortical pathways and cortical regions, the left brain hemisphere was harvested from a human cadaver and embalmed using a formaldehyde-based formula. The vessels, arachnoid, and pia mater membrane were carefully removed to expose the cortical surface. Photographs of the cortical surface were taken and processed with Adobe Illustrator (Adobe Systems Incorporated) to label the frontal operculum and overlay the image with different subcortical white-matter tracts.

\section{Statistical Analysis}

The chi-square test was used for all categorical data, with risk ratios (RRs) and 95\% confidence intervals (CIs) reported. The Student t-test was used for all continuous data. All statistical analysis was performed using SPSS (version 21, IBM Corp.).

\section{Results}

We identified 43 patients who met the inclusion criteria. Demographics are included in Table 1. Awake craniotomies with language mapping were performed in $74.4 \%$ of patients, while the remainder underwent craniotomies under general anesthesia. As previously described, any tumor greater than $3 \mathrm{~cm}$ from the prerolandic sulcus was operated on while the patient was asleep. Forty-one patients were right-handed, and 2 were left-handed. Both left-handed patients were confirmed as having left-sided language areas (1 using direct stimulation mapping and the other with preoperative MSI). The pars opercularis was the most frequently involved region (86.0\%), with fewer tumors involving the pars triangularis $(69.8 \%)$, and the least involving the pars orbitalis (32.6\%; Tables 2 and 3). Representative cases are depicted in Figs. 2 and 3.

Twenty-seven patients underwent preoperative MSI to 

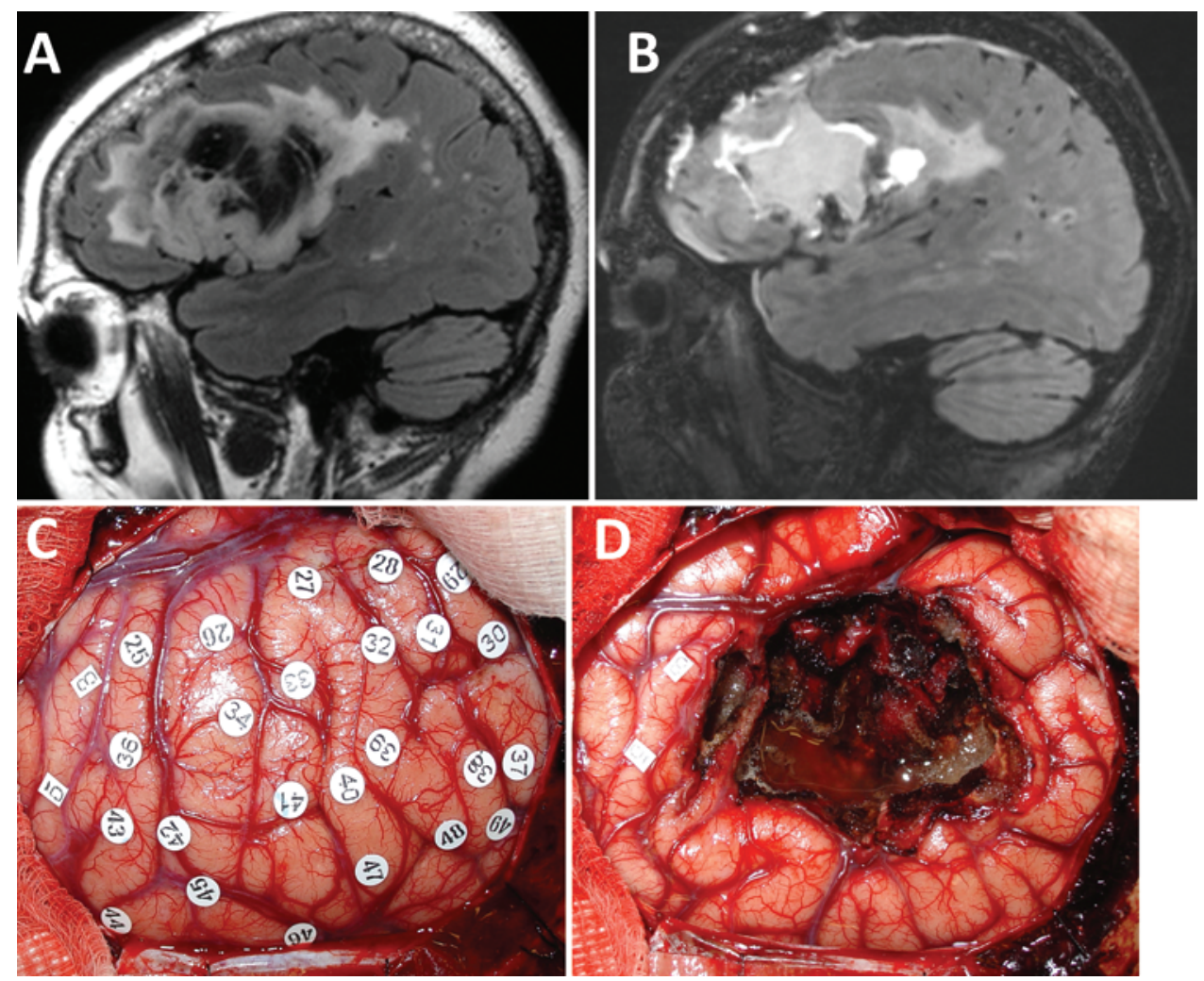

FIG. 3. Magnetic resonance images ( $A$ and $B$ ) and intraoperative photographs ( $C$ and $D)$ from a representative patient with postoperative language dysfunction. A: Preoperative parasagittal FLAIR image showing the patient's left frontal opercular oligoastrocytoma involving the pars triangularis and opercularis (but not orbitalis). B: Postoperative FLAIR image showing GTR of the tumor. The patient had transient difficulty with speech initiation postoperatively, which resolved completely within 2 weeks. C: Intraoperative photograph of the operculum prior to resection and after mapping. Stimulation of the area under tag 3 evoked tongue movement, and stimulation of area 5 evoked lip movement. No stimulation produced errors in naming or repetition. D: Intraoperative photograph after the resection was completed showing GTR, confirmed radiologically postoperatively. Figure is available in color online only.

localize language function. The majority of these images showed language function confined to the left frontal lobe (17 patients, 63.0\%), although many had bifrontal localization (7 patients, $25.9 \%)$. Two patients $(7.4 \%)$ had right frontal, along with left temporal and left parietal, representation. The remaining patient underwent MSI and had no sites identified. Whether patients had preoperative MSI was not predictive of speech dysfunction postoperatively (Table 4). Similarly, MSI localization of language (i.e., left frontal, bifrontal, etc.) was not significantly predictive of postoperative deficits (Table 4).

In the immediate postoperative period, 16 patients (37.2\%) had language deficits. Fifteen of these patients had tumors involving the pars opercularis, compared with only 2 involving the pars orbitalis and 12 the pars triangularis (Table 2). In 10 of these patients, their deficits resolved completely within 3 months of follow-up. Four (9.8\%) of 41 patients, however, had persistent deficits lasting longer than 3 months. Three of these 4 patients underwent awake language mapping, which identified language sites in only 1 patient (10 $\mathrm{mm}$ distant from the resection site). Diffusion-weighted imaging showed restricted diffusion in 2 of the 4 patients with persistent deficits, compared with 10 of 12 patients (83\%) who had diffusion restriction but only transient deficits. Two patients $(4.7 \%)$ were lost to followup (Table 2).

Of the 43 patients in the study, $7(16.3 \%)$ had preexisting language deficits preoperatively. Six of these 7 patients with preoperative language deficits continued to have deficits postoperatively, while the deficits of the other patient resolved immediately following surgery. For the 6 patients with both preoperative and postoperative language deficits, 2 had transient deficits (resolving within the first 3 months of follow-up), 3 had long-term deficits ( $>3$ months), and 1 was lost to follow-up.

All patient characteristics were compared for those patients with and without postoperative deficits in an attempt to find predictors of language morbidity (Table 4). Three factors were significantly associated with postoperative deficits. First, those patients presenting with seizures characterized by speech disturbances were significantly more likely to develop postoperative language deficits (RR 1.75, 95\% CI 1.02-2.98). Second, preoperative language deficits were highly predictive (RR 3.09, 95\% CI 1.68-5.66). Lastly, EOR, when quantitatively measured, was higher for patients without deficits than those with deficits $(95.1 \%$ $\pm 8.1 \%$ vs $87.7 \% \pm 14.6 \%$, respectively; $\mathrm{p}=0.04$ ). Also, though not statistically significant, increased age appeared 
TABLE 1. Patient demographics

\begin{tabular}{|c|c|}
\hline Characteristic & Value (\%) \\
\hline \multicolumn{2}{|l|}{ Age at surgery (yrs) } \\
\hline Mean & $42.6 \pm 12.5$ \\
\hline Median & 42 \\
\hline Range & $20-68$ \\
\hline \multicolumn{2}{|l|}{ Sex } \\
\hline Male & $26(60.5)$ \\
\hline Female & $17(39.5)$ \\
\hline \multicolumn{2}{|l|}{ Handedness } \\
\hline Rt & $41(95.3)$ \\
\hline Lt & $2(4.7)^{*}$ \\
\hline \multicolumn{2}{|l|}{ Tumor grade (WHO) } \\
\hline I & 0 \\
\hline II & $30(69.8)$ \\
\hline III & $6(14.0)$ \\
\hline IV & $7(16.3)$ \\
\hline \multicolumn{2}{|l|}{ Tumor pathology } \\
\hline Oligodendroglioma & $13(30.2)$ \\
\hline Oligoastrocytoma & $9(20.9)$ \\
\hline Astrocytoma & $8(18.6)$ \\
\hline Anaplastic oligodendroglioma & $2(4.7)$ \\
\hline Anaplastic oligoastrocytoma & $1(2.3)$ \\
\hline Anaplastic astrocytoma & $2(4.7)$ \\
\hline Glioblastoma & $7(16.3)$ \\
\hline Ependymoma & $1(2.3)$ \\
\hline \multicolumn{2}{|l|}{ Type of surgery } \\
\hline Awake & $32(74.4)$ \\
\hline General anesthesia & $11(25.6)$ \\
\hline \multicolumn{2}{|l|}{ Presentation } \\
\hline Seizures & $14(32.6)$ \\
\hline Seizures w/ aphasia & $10(23.3)$ \\
\hline Aphasia & $5(11.6)$ \\
\hline Headaches & $3(7.0)$ \\
\hline Sensorimotor disturbance & $3(7.0)$ \\
\hline Other & $9(20.9)$ \\
\hline
\end{tabular}

* Both left-handed patients were confirmed to have left-sided language representation.

somewhat predictive, with older patients more likely to develop postoperative language dysfunction $(\mathrm{p}=0.06)$. Tumor pathology, grade, patient sex, EOR when viewed dichotomously rather than quantitatively (i.e., GTR vs STR), diffusion changes postoperatively, and type of surgery appeared unrelated to postoperative deficits (Table 4).

We also determined whether the distance of intraoperatively mapped speech arrest sites to the resection cavity could affect patient outcomes. For the subset of patients where these data were available ( 29 patients, 13 with postoperative deficits and 16 without), we measured the distance of speech arrest sites, if present, from the margin of the resection cavity. Representative cases are depicted in Figs. 2 and 3. Nine patients (56\%) without postoperative deficits had no positive speech sites determined with intraoperative mapping, as compared with 8 patients $(62 \%)$ that did have postoperative language deficits (not significantly different; $p=0.78$ ). For those patients with documented speech arrest sites, the mean distance to the resection cavity was $5.6 \pm 2.4 \mathrm{~mm}$ for patients who did not have postoperative deficits compared with $6.8 \pm 4.1 \mathrm{~mm}$ for those patients who did have postoperative deficits, which again was not significantly different $(\mathrm{p}=0.53)$. There was no statistically significant difference in terms of language outcome between those patients who did and did not undergo mapping (RR for not mapping $=0.42,95 \%$ CI 0.11-1.55).

\section{Discussion}

The frontal operculum is known to harbor essential language regions and pathways. Because of these concerns, patients with tumors in this region are at a potentially higher risk for language morbidity postoperatively. Nevertheless, the exact risk to which these patients are exposed has yet to be delineated.

\section{Anatomy and Function}

The frontal operculum has been defined in multiple ways: as the combined pars orbitalis, triangularis, and opercularis, ${ }^{34}$ as the pars opercularis alone,${ }^{19}$ or as the areas ventral and medial to Brodmann's areas 44 and $45 .^{12}$ We have opted to choose the largest and most anatomically well-defined of these definitions, that of Yaşargil, and to further refine our results by the operculum's specific, welldelineated subregions: the pars orbitalis, pars triangularis, and pars opercularis (Fig. 1).

The dominant (usually left-sided) operculum deserves focus because of its historical importance and association with language. Interest was likely first concentrated on the operculum due to the findings of Pierre Broca, who described 2 patients, Leborgne and Lelong, both of whom had expressive aphasias and both of whom had lesions in the inferior frontal gyrus. ${ }^{10}$ This region went on to be termed Broca's area, and was further defined cytoarchi-

TABLE 2. Postoperative deficits and tumor location

\begin{tabular}{cccc}
\hline Tumor Location (any involvement) & No. of Patients & Patients w/ Transient Speech Deficit (\%) & Patients w/ Persistent Deficit (\%) \\
\hline Pars orbitalis & 14 & $2(14.3)$ & 0 \\
\hline Pars triangularis & 30 & $12(40.0)$ & $3 / 29(10.3)$ \\
\hline Pars opercularis & 37 & $15(40.5)$ & $4 / 35(11.4)$ \\
\hline Any frontal operculum & 43 & $16(37.2)$ & $4 / 41(9.8)$ \\
\hline
\end{tabular}

\footnotetext{
* Two patients lost to follow-up.
} 
TABLE 3. Postoperative deficits and specific tumor location

\begin{tabular}{lcc}
\hline \multicolumn{1}{c}{ Tumor Location } & $\begin{array}{c}\text { No. of } \\
\text { Patients }\end{array}$ & $\begin{array}{c}\text { Patients w/ } \\
\text { Speech Deficit (\%) }\end{array}$ \\
\hline Single region & & \\
\hline$\quad$ Pars orbitalis only & 2 & 0 \\
\hline Pars triangularis only & 1 & 0 \\
\hline$\quad$ Pars opercularis only & 11 & $4(36.4)$ \\
\hline Multiple regions & & \\
\hline Orbitalis + triangularis & 3 & $1(33.3)$ \\
\hline Triangularis + opercularis & 17 & $10(58.8)$ \\
\hline Orbitalis + opercularis & 0 & 0 \\
\hline $\begin{array}{l}\text { All 3 regions (orbitalis + triangula- } \\
\text { ris + opercularis) }\end{array}$ & 9 & $1(11.1)$ \\
\hline
\end{tabular}

tectonically as Brodmann's areas 44 and 45 (i.e., the pars opercularis and pars triangularis).

Interestingly, more recent studies have questioned the claim that injuries to Broca's area cause Broca's aphasias. Specifically, several studies have shown that Broca's aphasia is caused by lesions involving the insula, white matter underlying Broca's area, surrounding frontal cortex, basal ganglia, and superior temporal lobe, rather than Brodmann's areas 44 and 45 proper. ${ }^{10,20,25,26,32}$ Furthermore, the brains Broca examined himself have been reevaluated with CT and MRI, ${ }^{10}$ showing damage extending far beyond the inferior frontal gyrus: in the claustrum, putamen, caudate, globus pallidus, internal and external capsules, superior temporal lobe, the entire insula, and the inferior parietal lobe in Leborgne's brain, for example.

Critically, the area that appears to be most consistently damaged between the 2 specimens examined by Broca is the superior longitudinal fasciculus (SLF), which is a critical connection between anterior and posterior language areas (Fig. 4). ${ }^{10}$ This important tract is only now being anatomically delineated with intraoperative navigation systems using diffusion tensor imaging, and will likely become an ever more important surgical landmark as our techniques progress. In support of this evidence showing that areas aside from Broca's appear critically involved in expressive aphasias, there are also case reports of patients with lesions in Broca's area where speech is preserved. ${ }^{28}$

Given that it might not be critical for speech production, what does the frontal operculum contribute to speech and language? One hypothesis is that Brodmann's areas 44 and 45 (Broca's area) are important for verbal working memory, ${ }^{12,33}$ while other hypotheses include processing semantic content, ${ }^{13}$ syntax,,${ }^{14}$ and unification of multiple language subprocesses. ${ }^{16}$ It appears that no single task has been unanimously ascribed to these cortical areas. Importantly, however, many studies have shown that cortical stimulation can disrupt speech when applied to the inferior frontal gyrus, including Broca's area. ${ }^{29,31}$ But there is a high variability in the location of these speech arrest sites that cannot be definitively predicted by anatomical features. ${ }^{26}$ And in many patients (up to 40\%), no frontal lobe speech arrest sites can be found. ${ }^{31}$ Why some patients have clear speech arrest in Broca's area and others do not is unknown. This may be in part due to the patient population. Patients with tumors in the frontal operculum, those traditionally undergoing mapping, might undergo language reorganization with speech arrest sites disappearing from Broca's area to be relocated in nearby or even contralateral cortex..$^{11,22}$ Furthermore, the nature of mapping itself is unknown; that is, the exact neurophysiology of how stimulation affects language pathways continues to be actively investigated. . $^{1,18,30}$ Much more research is critically needed to understand not only the role of Broca's area in intact and tumor-affected brains, but also how our various methods of study (transcranial magnetic stimulation, MSI, functional MRI, electrical stimulation) relate to and affect language function.

The subcortical anatomy of the frontal operculum, especially the SLF and arcuate fasciculus (AF), appears critical to speech, ${ }^{10}$ although it is less well understood than the superficial cortical anatomy. Nevertheless, advances in diffusion tractography, improved dissection techniques in humans, and autoradiography studies in nonhuman primates are furthering our understanding of these important subcortical pathways. ${ }^{9,24}$ Classic white matter anatomical dissection shows the AF connecting the frontal operculum to the inferior parietal and superior temporal regions. ${ }^{10}$ A frontal-parietal segment of the $\mathrm{SLF}^{27}$ can also be isolated and exposed using advanced dissection techniques (Fig. 4A). ${ }^{24}$ However, with the development of advanced diffusion tractography - high angular resolution diffusion and diffusion spectrum imaging-these subcortical fascicles have been further studied in vivo in humans, which has provided evidence of a third component connecting the posterior temporal and inferior parietal regions (Fig. 4B) ${ }^{8}$ Nevertheless, autoradiography studies in nonhuman primates have recently provided conflicting results to the current understanding of the SLF and AF in humans. ${ }^{27}$ The autoradiography studies in the macaque monkey have provided evidence to subdivide the SLF into 4 fascicles in which the frontal operculum connects mainly to the parietal lobe via the AF and to the temporal lobe mainly via the extreme capsule (Fig. 4C). ${ }^{35}$ As our imaging and anatomical techniques continue to improve, along with our understanding of the overlying cortical function, we expect the nature of the subcortical areas of the operculum will become more clear, and our surgical approaches will undoubtedly benefit.

\section{Study Implications}

Our patient cohort included 43 patients with tumors involving the frontal operculum, 2 of whom were lost to follow-up. Twelve patients (27.9\%) had transient deficits in language function, while $4(9.8 \%)$ had persistent deficits. Several patient and operative characteristics appeared to predict postoperative language morbidity. Patient presentation with seizures characterized by speech disturbances and patients with preexisting language deficits were more likely to have postoperative language dysfunction than those patients with other presentations or without preoperative language deficits (RRs 1.75 and 3.09, respectively). It is important to note that seizures themselves were not predictive of poor outcome, but only seizures characterized by transient language dysfunction. Seizures provoking such speech dysfunction are perhaps a clue that the tumor is in close proximity to cortical regions critical for 
TABLE 4. Characteristics of patients with and without language deficits

\begin{tabular}{|c|c|c|c|}
\hline Characteristic & Patients (\%) w/o Deficit & Patients (\%) w/ Deficit & $\mathrm{RR}(95 \% \mathrm{Cl})$ or $p$ Value \\
\hline No. of patients & 27 & 16 & \\
\hline \multicolumn{4}{|l|}{ Age at surgery } \\
\hline Mean & $39.8 \pm 11.6$ & $47.2 \pm 13.0$ & $p=0.06$ \\
\hline Median & 40 & 49 & \\
\hline Range & $20-68$ & $23-65$ & \\
\hline \multicolumn{4}{|l|}{ Sex } \\
\hline Male & $18(66.7)$ & $8(50.0)$ & 1 (ref.) \\
\hline Female & $9(33.3)$ & $8(50.0)$ & $1.53(0.71-3.29)$ \\
\hline \multicolumn{4}{|l|}{ Tumor grade (WHO) } \\
\hline I & 0 & 0 & \\
\hline II & $18(66.7)$ & $12(75.0)$ & 1 (ref.) \\
\hline III & $5(18.5)$ & $1(6.3)$ & $0.42(0.07-2.63)$ \\
\hline IV & $4(14.8)$ & $3(18.8)$ & $1.07(0.41-2.80)$ \\
\hline \multicolumn{4}{|l|}{ Tumor pathology } \\
\hline Oligodendroglioma & $9(33.3)$ & $4(25.0)$ & 1 (ref.) \\
\hline Oligoastrocytoma & $5(18.5)$ & $4(25.0)$ & $1.44(0.48-4.32)$ \\
\hline Astrocytoma & $4(14.8)$ & $4(25.0)$ & $1.63(0.56-4.74)$ \\
\hline Anaplastic oligodendroglioma & $2(7.4)$ & 0 & $-{ }^{*}$ \\
\hline Anaplastic oligoastrocytoma & $1(3.7)$ & 0 & $-{ }^{*}$ \\
\hline Anaplastic astrocytoma & $1(3.7)$ & $1(6.3)$ & $-{ }^{*}$ \\
\hline Glioblastoma & $4(14.8)$ & $3(18.8)$ & $1.39(0.43-4.54)$ \\
\hline Ependymoma & $1(3.7)$ & 0 & $--^{*}$ \\
\hline \multicolumn{4}{|l|}{ Type of surgery } \\
\hline Awake w/ language mapping & $18(66.7)$ & $14(87.5)$ & 1 (ref.) \\
\hline General anesthesia & $9(33.3)$ & $2(12.5)$ & $0.42(0.11-1.55)$ \\
\hline \multicolumn{4}{|l|}{ Preop MSI } \\
\hline No & $13(48.1)$ & $3(18.8)$ & 1 (ref.) \\
\hline Yes & $14(51.9)$ & $13(81.3)$ & $2.56(0.86-7.66)$ \\
\hline Lt frontal & $7(50.0)$ & $10(76.9)$ & 1 (ref.) \\
\hline Bifrontal & $4(28.6)$ & $3(23.1)$ & $0.73(0.28-1.87)$ \\
\hline Rt frontal, It temporal, It parietal & $2(14.3)$ & 0 & $-{ }^{*}$ \\
\hline No sites identified & $1(7.1)$ & 0 & $-{ }^{*}$ \\
\hline \multicolumn{4}{|l|}{ EOR } \\
\hline GTR & $14(51.9)$ & $5(31.3)$ & 1 (ref.) \\
\hline STR & $13(48.1)$ & $11(68.8)$ & $1.36(0.58-3.19)$ \\
\hline \multicolumn{4}{|l|}{ Quantitative EOR } \\
\hline Mean overall percentage & $95.1 \pm 8.1$ & $87.7 \pm 14.6$ & $p=0.04 \dagger$ \\
\hline$\geq 90 \%$ & $22(81.5)$ & $9(56.3)$ & 1 (ref.) \\
\hline$\geq 80-90 \%$ & $4(14.8)$ & $3(18.8)$ & $1.48(0.53-4.08)$ \\
\hline$\geq 70-80 \%$ & 0 & $2(12.5)$ & $-{ }^{*}$ \\
\hline$\geq 60-70 \%$ & $1(3.7)$ & 0 & $-{ }^{*}$ \\
\hline$\geq 50-60 \%$ & 0 & $2(12.5)$ & $-{ }^{*}$ \\
\hline \multicolumn{4}{|l|}{ Diffusion restriction postop } \\
\hline No & $10(37.0)$ & $4(25.0)$ & 1 (ref.) \\
\hline Yes & $17(63.0)$ & $12(75.0)$ & $1.19(0.79-1.79)$ \\
\hline \multicolumn{4}{|l|}{ Presentation } \\
\hline Seizures & $11(40.7)$ & $3(18.8)$ & 1 (ref.) \\
\hline Seizures w/ speech disturbance & $2(7.4)$ & $8(50.0)$ & $1.75(1.02-2.98) \dagger$ \\
\hline Speech disturbance $\ddagger$ & $1(3.7)$ & $4(25.0)$ & $1.75(0.94-3.24)$ \\
\hline
\end{tabular}


TABLE 4. Characteristics of patients with and without language deficits (continued)

\begin{tabular}{|c|c|c|c|}
\hline Characteristic & Patients (\%) w/o Deficit & Patients (\%) w/ Deficit & RR $(95 \% \mathrm{Cl})$ or $p$ Value \\
\hline \multicolumn{4}{|l|}{ Presentation (continued) } \\
\hline Headaches & $3(11.1)$ & 0 & $-^{*}$ \\
\hline Sensorimotor disturbancef & $2(7.4)$ & $1(6.3)$ & $0.73(0.14-3.82)$ \\
\hline Other & $8(29.6)$ & $1(6.3)$ & $0.24(0.04-1.62)$ \\
\hline \multicolumn{4}{|l|}{ Preop language deficit } \\
\hline No & $26(96.3)$ & $10(62.5)$ & 1 (ref.) \\
\hline Yes & $1(3.7)$ & $6(37.5)$ & 3.09 (1.68-5.66)† \\
\hline
\end{tabular}

proper speech function, whereas seizures that do not affect speech might imply that the tumor is well separated from eloquent regions. Going forward, these patients should be advised that they might be at higher risk for transient speech disturbances postoperatively.

Another predictor of postoperative deficits appears to be the tumor EOR. When quantified, EOR differed significantly between patients with and without postoperative deficits $(87.7 \% \pm 14.6 \%$ vs $95.1 \% \pm 8.1 \%$, respectively; $p$ $=0.04)$. The finding that EOR is lower in patients with speech dysfunction is possibly due to more complicated tumors closer to functional regions or larger tumors, which might independently correlate with worse outcomes. However, when intraoperative mapping was analyzed, there was no significant difference between patients with speech arrest sites close to the resection cavity compared with those that were farther away, or not found at all. The absence of this finding may be attributable to the low sample size (29 patients). A prior study found that greater distance of the resection cavity to a positive speech site $(>1 \mathrm{~cm})$ reduced the risk of postoperative language deficits. ${ }^{15}$ However, this study evaluated only temporal lobe language sites, did not assess the operculum, and evaluated more patients with stimulation mapping (40, as compared with 29 in the current study). ${ }^{15}$

Preoperative MSI was performed in 27 patients as an adjunct method to lateralize language. The majority of patients had left-sided language (63.0\%), as expected by their hand dominance and the general frequency of left-sided language in the population. However, MSI frequently also found bifrontal or more dispersed language activation. MSI data were, however, not predictive of postoperative deficits (Table 4), although there was a trend among patients with right frontal language (2 patients), neither of whom developed postoperative language dysfunction. This lack of significant results is perhaps due to the small sample size (27 patients), but also possibly due to the limitations of MSI itself. For instance, Kim and Chung ${ }^{21}$ and Hirata et al. ${ }^{17}$ found concordance between MSI and Wada testing in patients of only $71 \%$ and $85 \%$, respectively. Such studies show that, while useful, MSI data cannot be relied on to unequivocally show language lateralization.

Lastly, tumors involving the pars orbitalis appear to be at particularly low risk for speech deficits, with no patients experiencing a permanent deficit and, for tumors isolated exclusively to this area, none experiencing a transient deficit.

Overall, there was a low risk for permanent speech deficits in patients undergoing resection of the frontal operculum, which occurred in only 4 patients $(9.8 \%)$. This is consistent with prior reports that showed limited or no morbidity in small numbers of patients. ${ }^{23,28}$ The majority of patients with permanent deficits $(n=3)$ had preexisting speech dysfunction prior to surgery, yielding a risk of $2.9 \%$ of permanent deficit in those patients without dysfunction preoperatively. Gross-total resection of operculum tumors was possible in more than $40 \%$ of patients, and patients with larger EORs appeared to have improved language outcomes. This observed low morbidity rate and the high degree of resection support the inclusion of resection in the overall management of patients with operculum gliomas. However, further study is warranted to determine the neuropsychiatric effects of surgery in this area, in addition to the effect on overall survival and quality of life.

\section{Limitations of the Study}

There are several limitations to the current study. First, this is a retrospective review of patients. Second, there is a probable bias in patient selection. There are likely many patients with left frontal tumors who are not referred to us if their disease is particularly advanced, and our study does not include patients with left frontal tumors that were deemed by others to not be surgical candidates. Third, detailed neuropsychological testing was not conducted in our patients. We attempted to be as liberal as possible with our definition of language dysfunction, so as to identify patients with even subtle deficits. However, we might still be missing more deficits that can only be documented on pre- and postoperative testing. This last point will hopefully be addressed in further studies that continue to assess the role of surgery in patients with tumors in this important location.

\section{Conclusions}

Despite the presumed importance of the frontal operculum in speech production, the outcome of tumor resection in this region has been underreported. We therefore analyzed our cohort of 43 patients undergoing resection of left frontal operculum tumors: $27.9 \%$ of patients expe- 

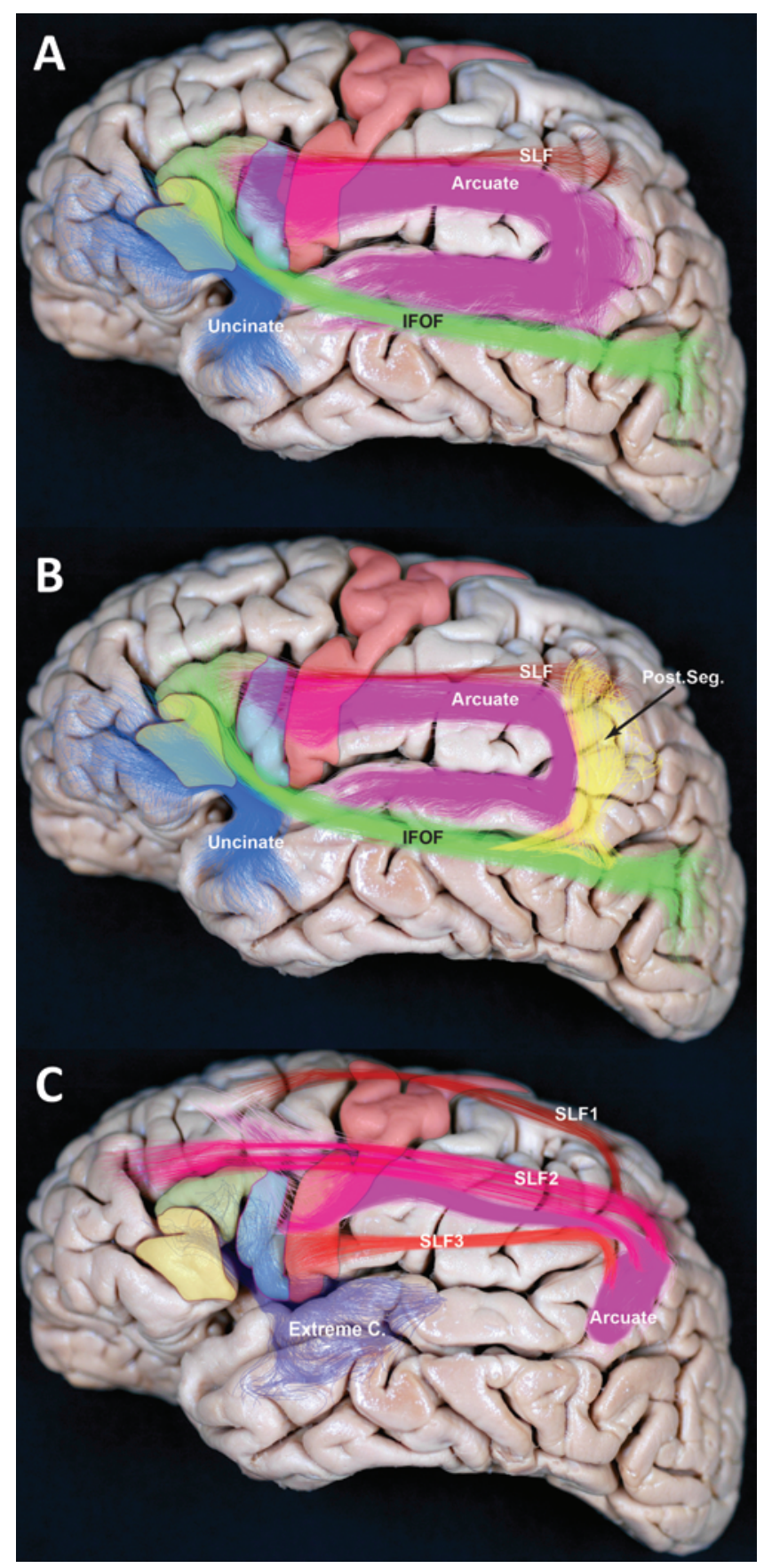

FIG. 4. Subcortical pathways of the SLF and AF. A: This lateral view of a cadaveric brain shows the frontal operculum, along with several components of the SLF and AF. Solid shading indicates anatomical areas: pars orbitalis (yellow), pars triangularis (green), pars opercularis (blue), and motor cortex (red). Fiber pathways are indicated with colored bundles: SLF (red), AF (purple), inferior frontal-occipital fasciculus (IFOF; green), and uncinate fasciculus (blue). B: Areas and representative colors are the same as in the previous panel, but also shown is the posterior pathway (post. seg.) connecting posterior temporal and inferior parietal regions (yellow). C: SLF fascicles, as identified using autoradiography in the macaque monkey, are projected into the human brain. The SLF is believed to be divided into the 3 segments (SLF1, SLF2, and SLF3), along with the AF. Only SLF3 and the AF connect to the frontal operculum. Extreme C. = extreme capsule. rienced transient deficits, and 9.8\% had long-term language dysfunction postoperatively $(2.9 \%$ when those patients with preoperative language dysfunction were excluded). Risk factors of postoperative dysfunction appear to be preoperative language deficits, along with seizures characterized by language deficits. Patients with tumors confined to the pars orbitalis alone have no risk for postoperative language dysfunction following resection. Given the importance of maximal safe resection in the overall outcome of individuals with gliomas, and given the low rate of language morbidity presented here, resection of intrinsic tumors involving the frontal operculum should be considered in these patients.

\section{References}

1. Agnesi F, Johnson MD, Vitek JL: Deep brain stimulation: how does it work? Handb Clin Neurol 116:39-54, 2013

2. Alexander MP, Naeser MA, Palumbo C: Broca's area aphasias: aphasia after lesions including the frontal operculum. Neurology 40:353-362, 1990

3. Amunts K, Zilles K: Architecture and organizational principles of Broca's region. Trends Cogn Sci 16:418-426, 2012

4. Berger MS, Cohen WA, Ojemann GA: Correlation of motor cortex brain mapping data with magnetic resonance imaging. J Neurosurg 72:383-387, 1990

5. Berger MS, Ojemann GA: Intraoperative brain mapping techniques in neuro-oncology. Stereotact Funct Neurosurg 58:153-161, 1992

6. Berger MS, Ojemann GA, Lettich E: Neurophysiological monitoring during astrocy toma surgery. Neurosurg $\mathbf{C l i n} \mathbf{N}$ Am 1:65-80, 1990

7. Burns MS, Fahy J: Broca's area: rethinking classical concepts from a neuroscience perspective. Top Stroke Rehabil 17:401-410, 2010

8. Catani M, Jones DK, ffytche DH: Perisylvian language networks of the human brain. Ann Neurol 57:8-16, 2005

9. Dick AS, Tremblay P: Beyond the arcuate fasciculus: consensus and controversy in the connectional anatomy of language. Brain 135:3529-3550, 2012

10. Dronkers NF, Plaisant O, Iba-Zizen MT, Cabanis EA: Paul Broca's historic cases: high resolution MR imaging of the brains of Leborgne and Lelong. Brain 130:1432-1441, 2007

11. Duffau H: The huge plastic potential of adult brain and the role of connectomics: new insights provided by serial mappings in glioma surgery. Cortex 58:325-337, 2014

12. Friederici AD: The brain basis of language processing: from structure to function. Physiol Rev 91:1357-1392, 2011

13. Friederici AD: Towards a neural basis of auditory sentence processing. Trends Cogn Sci 6:78-84, 2002

14. Grodzinsky Y: The neurology of syntax: language use without Broca's area. Behav Brain Sci 23:1-71, 2000

15. Haglund MM, Berger MS, Shamseldin M, Lettich E, Ojemann GA: Cortical localization of temporal lobe language sites in patients with gliomas. Neurosurgery 34:567-576, 1994

16. Hagoort P: On Broca, brain, and binding: a new framework. Trends Cogn Sci 9:416-423, 2005

17. Hirata M, Goto T, Barnes G, Umekawa Y, Yanagisawa T, Kato A, et al: Language dominance and mapping based on neuromagnetic oscillatory changes: comparison with invasive procedures. J Neurosurg 112:528-538, 2010

18. Histed MH, Bonin V, Reid RC: Direct activation of sparse, distributed populations of cortical neurons by electrical microstimulation. Neuron 63:508-522, 2009

19. Keller SS, Roberts N, Hopkins W: A comparative magnetic resonance imaging study of the anatomy, variability, and asymmetry of Broca's area in the human and chimpanzee brain. J Neurosci 29:14607-14616, 2009 
20. Kertesz A, Harlock W, Coates R: Computer tomographic localization, lesion size, and prognosis in aphasia and nonverbal impairment. Brain Lang 8:34-50, 1979

21. Kim JS, Chung CK: Language lateralization using MEG beta frequency desynchronization during auditory oddball stimulation with one-syllable words. Neuroimage 42:1499-1507, 2008

22. Kośla K, Pfajfer L, Bryszewski B, Jaskólski D, Stefańczyk L, Majos A: Functional rearrangement of language areas in patients with tumors of the central nervous system using functional magnetic resonance imaging. Pol J Radiol 77:39-45, 2012

23. Kral T, Kurthen M, Schramm J, Urbach H, Meyer B: Stimulation mapping via implanted grid electrodes prior to surgery for gliomas in highly eloquent cortex. Neurosurgery 58 (1 Suppl):ONS36-ONS43, 2006

24. Martino J, De Witt Hamer PC, Vergani F, Brogna C, de Lucas EM, Vázquez-Barquero A, et al: Cortex-sparing fiber dissection: an improved method for the study of white matter anatomy in the human brain. J Anat 219:531-541, 2011

25. Mohr JP, Pessin MS, Finkelstein S, Funkenstein HH, Duncan GW, Davis KR: Broca aphasia: pathologic and clinical. Neurology 28:311-324, 1978

26. Naeser MA, Hayward RW: Lesion localization in aphasia with cranial computed tomography and the Boston Diagnostic Aphasia Exam. Neurology 28:545-551, 1978

27. Petrides M, Pandya DN: Distinct parietal and temporal pathways to the homologues of Broca's area in the monkey. PLoS Biol 7:e1000170, 2009

28. Plaza M, Gatignol P, Leroy M, Duffau H: Speaking without Broca's area after tumor resection. Neurocase 15:294-310, 2009

29. Quiñones-Hinojosa A, Ojemann SG, Sanai N, Dillon WP, Berger MS: Preoperative correlation of intraoperative cortical mapping with magnetic resonance imaging landmarks to predict localization of the Broca area. J Neurosurg 99:311-318, 2003
30. Rolston JD, Desai SA, Laxpati NG, Gross RE: Electrical stimulation for epilepsy: experimental approaches. Neurosurg Clin N Am 22:425-442, v, 2011

31. Sanai N, Mirzadeh Z, Berger MS: Functional outcome after language mapping for glioma resection. N Engl J Med 358: $18-27,2008$

32. Schiff HB, Alexander MP, Naeser MA, Galaburda AM: Aphemia. Clinical-anatomic correlations. Arch Neurol 40:720-727, 1983

33. Smith EE, Jonides J: Storage and executive processes in the frontal lobes. Science 283:1657-1661, 1999

34. Yaşargil MG, Curcic M, Abernathey CD: Microneurosurgery: Microneurosurgery of CNS Tumors. New York: Thieme, Vol IV B, 1996

35. Yeterian EH, Pandya DN, Tomaiuolo F, Petrides M: The cortical connectivity of the prefrontal cortex in the monkey brain. Cortex 48:58-81, 2012

\section{Author Contributions}

Conception and design: Rolston, Berger. Acquisition of data: Rolston, Benet, Li, Berger. Analysis and interpretation of data: Rolston, Englot, Benet, Cha, Berger. Drafting the article: Rolston. Critically revising the article: Rolston, Englot, Benet, Berger. Reviewed submitted version of manuscript: all authors. Approved the final version of the manuscript on behalf of all authors: Rolston. Statistical analysis: Rolston. Administrative/technical/ material support: Berger. Study supervision: Berger.

\section{Correspondence}

John D. Rolston, 505 Parnassus Ave., M779, San Francisco, CA 94143-0112. email: rolstonj@neurosurg.ucsf.edu. 